\title{
Temporal references in dreams and autobiographical memory
}

\author{
JEAN GRENIER, PHILIPPE CAPPELIEZ, MÉLANIE ST-ONGE, JULIE VACHON, \\ SOPHIE VINETTE, FRANCINE ROUSSY, PIERRE MERCIER, \\ MONIQUE LORTIE-LUSSIER, and JOSEPH DE KONINCK \\ University of Ottawa, Ottawa, Ontario, Canada
}

\begin{abstract}
In an attempt to determine whether temporal references identified in dreams follow the same temporal distributions as those documented for autobiographical memories, 28 younger women (18-35 years of age) and 30 older women (60-77 years of age) kept a home dream diary for 1 week and then slept 1 night in the laboratory for rapid eye movement sleep dream collection. The following morning, they identified temporal references in their dreams and produced a sample of autobiographical memories using the semantic cuing method. For both groups, there was a linear decrease in temporal references identified in dreams and autobiographical memories with increased remoteness for the last 30 years. As predicted, for the older group, there were similar cubic trends reflecting a disproportionately higher number of both temporal references identified in dreams and autobiographical memories from adolescence/ early adulthood compared with adulthood and childhood. The results support the notion of continuity between waking and dreaming memory processes.
\end{abstract}

Several authors have discussed how dream processes are related in some way to cognitive and memory processes (Cavallero \& Foulkes, 1993; Hobson, 1988). Most notably, Foulkes (1985) stated that dream production mechanisms draw bits and pieces of elements within the vast mnemonic repertoire that contains all the information, knowledge, and personal memories accumulated over an entire lifetime. He further postulated that the cognitive organization of dreams should reflect that of the waking state. It would thus follow that any pattern by which temporal references would be included in dreams would parallel that of autobiographical memory, which has been studied and modeled quite systematically.

Indeed, Rubin, Wetzler, and Nebes (1986) integrated data from four studies on autobiographical memory (Fitzgerald \& Lawrence, 1984; Franklin \& Holding, 1977; Rubin et al., 1986; Zola-Morgan, Cohen, \& Squire, 1983) and elaborated a three-component model to describe the distribution of autobiographical memories across the lifespan. The first component is a retention function referring to the general finding that it is increasingly difficult to recall memories as they become more and more remote. This retention function describes the entire distribution of autobiographical memories recalled by young adults (under 35 years of age), and for the most recent 30 years in the case of middle-aged and older adults.

Support for this research was provided by the Natural Sciences and Engineering Research Council of Canada. Correspondence should be addressed to J. De Koninck, School of Psychology, University of Ottawa, P.O. Box 450, Station A, Ottawa, ON, K1N 6N5 Canada (e-mail: jdekonin@uottawa.ca).
The second component of the model refers to a reminiscence process; that is, adults over the age of 40 have a tendency to recall life experiences dating back to adolescence and/or early adulthood. This component of the model is meant to explain the disproportionate amount of memories of events and activities that originally took place when a person was between 10 and 30 years olda phenomenon often referred to as a differential resurgence or reminiscence bump of memories. Childhood amnesia constitutes the third component of the model: People of all ages rarely remember events that took place before they were 6 years old.

It was of great interest to determine whether references to past events in dreams share the same characteristics. The existing literature provides very limited information. First, several studies have concluded that a significant proportion of dreams contain elements that refer to experiences of the preceding day, a phenomenon known as day residues (Botman \& Crovitz, 1989; Davidson \& Kelsey, 1987; Epstein, 1985; Hartmann, 1968; Jouvet, 1979; Marquardt, Bonato, \& Hoffman, 1996; Nielsen \& Powell, 1992; Verdone, 1965), whereas other studies have focused on dream elements that stem from experiences that took place 1 week prior to the dream, a phenomenon called the dream-lag effect (Nielsen \& Powell, 1992). Verdone's laboratory study was the first to systematically identify temporal references in dreams that were related to experiences from a remote past - that is, over 5 years prior to the dream. But, so far, only one study (Botman \& Crovitz, 1989) has examined the continuity of dreaming with autobiographical memory. They used a questionnaire to ask participants to identify (1) temporal references in a dream from the previous night, (2) a most 
vivid dream, and (3) a vivid memory of a past event. Observing that the distribution of memories of dreams followed closely that of life events, they concluded that autobiographical memory continues to function during sleep. In other words, recall diminishes with time. However, the method used for collecting data was limited to only one dream and one event, thus simply concentrating on the capacity of young adults to recall a dream and its date of occurrence.

The question therefore remains: Is the temporal organization of mnemonic sources to which dreams refer during sleep the same as the organization observed for autobiographical memory during wakefulness? We considered that the model of Rubin et al. (1986) could be used to study the temporal distribution of elements identified in dreams. From this perspective, we predicted that in young adults, the distribution of dream temporal references, as well as that of autobiographical memories, would follow a monotonically decreasing power function. There should be a high frequency of recent temporal references, decreasing with increasing remoteness (retention function component), ending with a paucity of temporal references dating back to early childhood (childhood amnesia component). For older adults, we predicted that the distribution of dream temporal references, as well as the distribution of autobiographical memories, would follow a cubic trend. First, there would be a high frequency of temporal references dating back to the last 10-20 years, decreasing with increasing remoteness until approximately 30 years ago (retention function component). It would be followed by a resurgence of temporal references to experiences dating back to adolescence and/or young adulthood (reminiscence component). Finally, as with young adults, there would be a paucity of temporal references to experiences that took place during early childhood (childhood amnesia component).

To rectify some of the limitations of Botman and Crovitz's (1989) study, the continuity of autobiographical memory during sleep was examined through systematic analysis of the distribution of temporal references identified in dreams collected via the home dream diary method as well as via rapid eye movement (REM) awakenings in a sleep laboratory. Since each method has its limitations, and given the controversy regarding their respective value (Domhoff, 2003), obtaining dreams with both procedures would ensure a more comprehensive sampling of potential temporal references and better acceptance of the findings.

\section{METHOD}

\section{Participants}

A total of 58 women participated in this study: 28 undergraduate students (age range $=18-35 ; M=22$ ) and 30 retired teachers and nurses (age range $=60-77 ; M=65$ ). Only women were invited to participate in order to eliminate gender as a source of variability. In addition, we know from several studies (Giambra, Jung, \& Grodsky, 1996) that women tend to have better dream recall than men do. Because education and socioeconomic levels influence dream content (Winget, Kramer, \& Whitman, 1970), participants in the two age groups were matched on these two dimensions. The older participants were recruited from the Ottawa area; the young participants were recruited from the pool of undergraduate psychology students at the University of Ottawa. After completing a general information questionnaire (demographics, medication, etc.), the participants were evaluated with the Modified Mini-Mental Status Examination (3-MS; Stuss, Meiran, Guzman, Lafleche, \& Willmer, 1996) and the Beck Depression Inventory (Beck, Ward, Mendleson, Mock, \& Erbaugh, 1961). The inclusion criteria were (1) intact cognitive functioning and (2) ability to recall dreams upon awakening in the morning. The exclusion criteria were (1) presence of clinically significant depression, (2) sleep disorders or sleep difficulties, or (3) psychotropic medication or use of hypnotics.

Mean scores on the 3-MS examination for the younger and older groups were 99 and 98 , respectively, with all participants obtaining scores equal to or above 97, indicative of intact cognitive functioning. As for the Beck Depression Inventory, 7 participants obtained scores within the mild-to-moderate depression categories (between 11 and 18). They were retained, however, because they were not taking any antidepressant medication and their sleep architecture (the organization of sleep stages as defined by sleep researchers) was normal (documented polysomnographically).

\section{Design and Procedure}

After the initial telephone interview, the procedure unfolded this way: (1) The participants took a guided tour of the laboratory facilities to familiarize themselves with the environment and to complete the assessment; (2) they kept a home dream diary for a week in which dreams were to be recorded upon awakening; (3) they then slept 1 night in the laboratory where they were awakened in all REM periods and asked to report their mental experience; (4) in the morning, upon awakening in the laboratory, they were interviewed to identify temporal references in their laboratory and home dreams; and (5) before leaving the laboratory, they generated a sample of autobiographical memories using the semantic cuing method. Scheduling was alternated between age groups to minimize any order effect.

\section{Dream Collection}

The home dream collection was carried out during the week immediately preceding the night in the laboratory. The participants were instructed to write in the morning, with as many details as possible, their recall of a dream immediately following awakening each day of the week.

As for dreams in the laboratory, awakenings for REM dream reports were made 5 min after the first REM burst of the initial REM period and 10 min after the first REM burst of each successive REM period. The identification of sleep stages was conducted as per Rechtschaffen and Kales (1968). REM awakenings were conducted by four laboratory assistants (honor students in psychology) blind to the hypotheses. Each participant interacted with the same assistant during REM awakenings and the morning interview. The participant, sleeping alone in a room, heard her name called via an intercom and was then asked: "What was going through your mind at the moment you were awakened?" After a few seconds of silence, the assistant repeated the participant's last sentence and/or asked if there were any more details to be added. All REM dream reports were audiotaped and immediately transcribed by the laboratory assistants. The participants went to bed at their usual time (between 22:00 and 24:00). The final morning awakening was carried out between 6:00 and 7:00.

\section{Morning Interview}

To limit the morning interview to a maximum of $3 \mathrm{~h}$, no more than four REM dreams and three home dreams were randomly selected. Once dreams were selected, the assistants segmented each dream into thematic units and identified every single character, setting, object, event, and activity. After each assistant had completed 
this task individually, they met together to counterverify the exactitude of the elements identified. To ensure methodological consistency, discrepancies were discussed until an agreement was reached. Dream elements were classified in five categories: characters (individuals, groups of individuals, animals, and creatures); settings (geographically identified regions, interior as well as exterior settings); objects (elements ordinarily recognized as objects such as a chair, parts of the anatomy, buildings, trees, etc.); events (everything that happens in the dream but is not inherent to the actions carried out by the dreamer or any other character, such as a wedding, an anniversary, the fact that it is raining, emotions explicitly described in the dream such as being afraid, etc.); and activities (physical or mental actions executed by the dreamer or other characters).

In the morning, the participants were interviewed by an assistant to identify temporal references in their home and laboratory dreams. This interview was conducted in the participant's room and was tape recorded. The assistant read the dream report aloud twice while the participant viewed a written copy. The assistant then proceeded to read each individual thematic unit and, for every dream element, the participant was asked:

(1) "Do you recognize the element in question?" The element was considered as is when the participant explained that, to the best of her judgment, she recognized the element as she had seen it in reality. Alternatively, it was considered generic if the participant said she could not recognize it. This as is/generic distinction was useful because dreaming sometimes produces images (i.e., characters, settings) that are faithful replicas of reality, and at other times, images that are distorted and unrecognizable. It was important to treat dream elements in a fashion congruent with this phenomenon. The as is/generic distinction is similar to the remember/know distinction in the literature. For example, in recognition-memory experiments, Gardiner and Java (1991) asked subjects to indicate when recognizing a word whether its recognition was accompanied by some recollective experience (remember) or whether it was recognized on the basis of familiarity without any recollective experience (know).

(2) "When was the last time you saw or experienced the element in question (a person, object, setting, or experienced an activity or event)?" If the element was considered as is, the assistant referred to it as it appeared in the dream. When a character in a dream appeared younger than in reality, the question was formulated as a function of the character's appearance as present in the dream (in a younger appearance). This logic and methodology were also applied to the other dream elements considered as is. If the element was considered generic, the assistant referred to the element in generic terms and not as it appeared in the dream.

For each of the identified elements, the participants were asked to identify temporal references in terms of two temporal scales. The first was in terms of "time ago" using the following 17 categories: this evening; yesterday; before yesterday; this week; a week ago; a month ago; 1-6 months ago; 7-11 months ago; $1-5$ years ago; 6-9 years ago; 10-19 years ago; 20-29 years ago; 30-39 years ago; 40-49 years ago; 50-59 years ago; 60-69 years ago; and 70-79 years ago. The second was in terms of the participant's reported "age at time of original experience" (or time of the event being encoded) using the following 8 categories: 70-79 years old; 60-69 years old; 50-59 years old; $40-49$ years old; $30-39$ years old; $20-29$ years old; 10-19 years old; and less than 10 years old. The use of these two temporal scales was agreed upon because some of the planned analyses of the data called for "time ago" indices, whereas others called for "age at time of original experience" indices.

(3) "Have you thought or talked about the element in question since you last saw or experienced it in reality?"

It should be noted that for both home and laboratory dreams, only temporal references that the participants identified as not having been accessed with conscious awareness since the time of the original experience were classified in temporal bins and analyzed. This was to correct for methodological limitations of earlier studies (i.e., Verdone, 1965) because one may have dreamed of a person who was last seen 25 years ago, but if one has thought or talked about this person the day prior to the dream, that day-old thought or discussion may have served as the mnemonic source for the elaboration of the dream. We recognize, however, that this methodology could work against finding a resurgence of memories in the remote past.

\section{Autobiographical Memories}

Finally, the participants were asked to produce a sample of autobiographical memories using the semantic cuing method. This task was scheduled in the morning to prevent contamination of dream content and to make sure it was performed at the same time of day for each participant. Twenty-five words individually typed on cards were randomly presented. The participants were asked to verbalize the first memory of a personal experience that came to mind. Two words (book, cotton) (McCormack, 1979) were utilized for practice; 25 other words randomly chosen from a list of 925 nouns (Paivio, Yuille, \& Madigan, 1968) were used for the experiment.

The 25 words or semantic stimuli were classified using the same five categories as for dream elements: characters (butcher, priest, musician, police, king); objects (chair, window, tree, blood, star); settings (church, street, house, forest, basement); events (ceremony, party, disaster, fire, Christmas); and activities (gardening, shopping, orchestrating, photographing, singing). These words were chosen for their familiar character and because they were classified by Paivio et al. (1968) as being high in the following characteristics: (1) concreteness (capacity to evoke experiences felt by the senses), (2) imagery (capacity to evoke nonverbal images), and (3) meaningfulness (capacity to facilitate quick retrieval of many associations). Autobiographical memory data collection was tape recorded. Once a memory was verbalized for every word, the tape was replayed, and the participant was asked to date each memory (standard dating method), in terms of the same two temporal scales used for dreams (see above).

\section{RESULTS}

\section{Descriptive Characteristics of Memories and Dreams for Both Age Groups}

Autobiographical memories. Older participants generated and dated 742 memories; younger participants generated and dated 696 memories.

Laboratory dreams. One hundred sixty-three REM dreams were obtained ( 91 from the younger participants and 72 from the older participants). For each participant, the percentage of dream recall was calculated by dividing the number of times a dream was recalled by the total number of REM awakenings. Younger participants recalled dreams significantly more often $(M=.98, S D=$ $.08)$ than did older participants $[M=.81, S D=.23$, $t(365.92)=-3.66, p<.01]$. The length (word count) of REM dreams was calculated on spontaneous dream reports (before the participant was asked whether there were any more details to add). Analyses indicated that the REM dreams of the younger participants were longer $(M=100.56, S D=70.23)$ than were those of the older participants $[M=63.79, S D=68.75, t(142)=-3.15$, $p<.01]$.

Using the selection processes explained previously, the minimum/maximum/mean numbers of REM dreams for the younger group was 2,4 , and 3 per participant, respectively. For the older group, the minimum/maxi- 
mum/mean numbers of REM dreams were 1,4 , and 2 per participant, respectively. In this way, temporal references were identified for 148 REM dreams ( 80 for the younger participants and 68 for the older participants). The distribution of these 148 dreams across REM periods is presented in Table 1. Only the temporal references that met the inclusion criteria previously described were classified and analyzed. The younger participants identified 661 references that met these criteria, whereas the older participants identified 474.

Home dreams. The younger participants reported a total of 110 home dreams and the older participants 145 . The length (word count) of home dreams of the younger participants did not differ $(M=160.71, S D=99.22)$ from those of the older participants $[M=141.03, S D=$ 94.29, $t(171)=-1.34, p<.05]$.

A total of 173 home dreams ( 82 and 91 dreams for the younger and older participants, respectively), randomly chosen, were retained for identification of temporal references. This represented a minimum $/$ maximum $/$ mean number of 2, 3, and 3 home dreams per younger participant and that of 2, 4, and 3 home dreams per older participant. Temporal references were identified for the 173 home dreams. The younger participants identified 743 references that met the inclusion criteria; the older participants identified 950 .

\section{Temporal References for Dreams and Autobiographical Memories of the Younger Participants}

For the younger adults, it had been predicted that the distribution of temporal references for both dreams and memories would follow a monotonically decreasing power function - that is, a high frequency of recent temporal references decreasing with increasing remoteness. In addition, the model predicted that young adults would show a paucity of temporal references dating back to early childhood.

From a descriptive point of view, the younger participants identified temporal references across all 17 "time ago" temporal categories for both laboratory and home dreams as well as for autobiographical memories. Memories contained significantly fewer references to "this evening" $(M=9.14, S D=9.67)$ than did REM dreams $[M=25.10, S D=14.79, t(27)=4.99, p<.01]$. Memories also contained significantly fewer references to "yesterday" $(M=8.29, S D=8.29)$ than did REM dreams $[M=16.81, S D=14.15, t(27)=3.15, p<.01]$. Home dreams contained significantly more references to "this evening" $(M=18.15, S D=18.49)$ than did memories $[M=9.14, S D=9.67, t(27)=2.35, p<.03]$.

To test the prediction of a monotonically decreasing power function, we used logarithmic scales because this procedure had been used in past work (Rubin, 1982; Rubin et al., 1986). We thus converted, for both memories and dreams, the number of temporal references per "time ago" bin to the number of temporal references per hour by dividing the number of temporal references by the
Table 1

Distribution of Analyzed Dreams and Temporal References Across REM Periods for Younger and Older Participants

\begin{tabular}{|c|c|c|c|c|c|c|c|c|}
\hline \multirow[b]{3}{*}{ REM } & \multicolumn{4}{|c|}{ Younger } & \multicolumn{4}{|c|}{ Older } \\
\hline & \multicolumn{2}{|c|}{ Dreams } & \multicolumn{2}{|c|}{$\begin{array}{c}\text { Temporal } \\
\text { References }\end{array}$} & \multicolumn{2}{|c|}{ Dreams } & \multicolumn{2}{|c|}{$\begin{array}{c}\text { Temporal } \\
\text { References }\end{array}$} \\
\hline & $n$ & $\%$ & $n$ & $\%$ & $n$ & $\%$ & $n$ & $\%$ \\
\hline 1 & 7 & 8.8 & 35 & 5.3 & 12 & 17.6 & 31 & 6.5 \\
\hline 2 & 24 & 30.0 & 162 & 24.5 & 24 & 35.3 & 157 & 33.1 \\
\hline 3 & 26 & 32.5 & 226 & 34.2 & 21 & 30.9 & 181 & 38.2 \\
\hline 4 & 15 & 18.7 & 177 & 26.7 & 9 & 13.2 & 89 & 18.8 \\
\hline 5 & 7 & 8.8 & 58 & 8.8 & 1 & 1.5 & 1 & 0.23 \\
\hline 6 & 1 & 1.2 & 3 & 0.5 & 1 & 1.5 & 15 & 3.17 \\
\hline Total & 80 & 100 & 661 & 100 & 68 & 100 & 474 & 100 \\
\hline
\end{tabular}

hours in each bin. This generated a $y$ value. The $x$ value was the midpoint of each bin, expressed in hours. A regression predicting the log of the $y$ values from the log of the $x$ values then provided a goodness of fit and slope parameter. Figure 1 is a plot of the log of temporal bin frequency as a function of the log of the bin marked in hours ago for memories, REM dreams, and home dreams of the younger participants. In all three cases, the distributions are decreasing with increasing remoteness, with slopes of $-0.14,-0.42$, and -0.17 and intercepts of 4.94, 6.42, and 5.22 for the memories, REM dreams, and home dreams, respectively.

As predicted, the young adults identified very few temporal references dating back to early childhood in both memories and in dreams: $5.46 \%$ and $0.86 \%$ in categories "10-19 years ago" and "20-29 years ago" for memories, $0.76 \%$ and $0.15 \%$ in categories " $10-19$ years ago" and "20-29 years ago" for REM dreams, and finally, $1.08 \%$ and $0.00 \%$ in the same categories for home dreams.

\section{Temporal References for Dreams and Autobiographical Memories of the Older Participants}

For the older adults, it had been predicted that the distribution of temporal references for both dreams and memories would follow a cubic trend, starting with a high frequency for recent temporal references, decreasing until a resurgence at young adulthood and adolescence, and ending with a paucity of early childhood references. These predictions were confirmed for autobiographical memories, as well as for dreams (laboratory and home dream data combined).

From a descriptive point of view for both memories and dreams, the older adults identified references across all "time ago" temporal categories. When compared with REM dreams $[M=16.77, S D=15.42, t(29)=4.96$, $p<.01]$ and with home dreams $[M=15.03, S D=$ $12.65, t(29)=5.18, p<.01]$, autobiographical memories contained fewer references $(M=4.00, S D=5.25)$ to "this evening." REM dreams also contained significantly more references to "yesterday" $[M=16.09, S D=$ 24.95, $t(29)=2.06, p<.05]$ compared with memories 

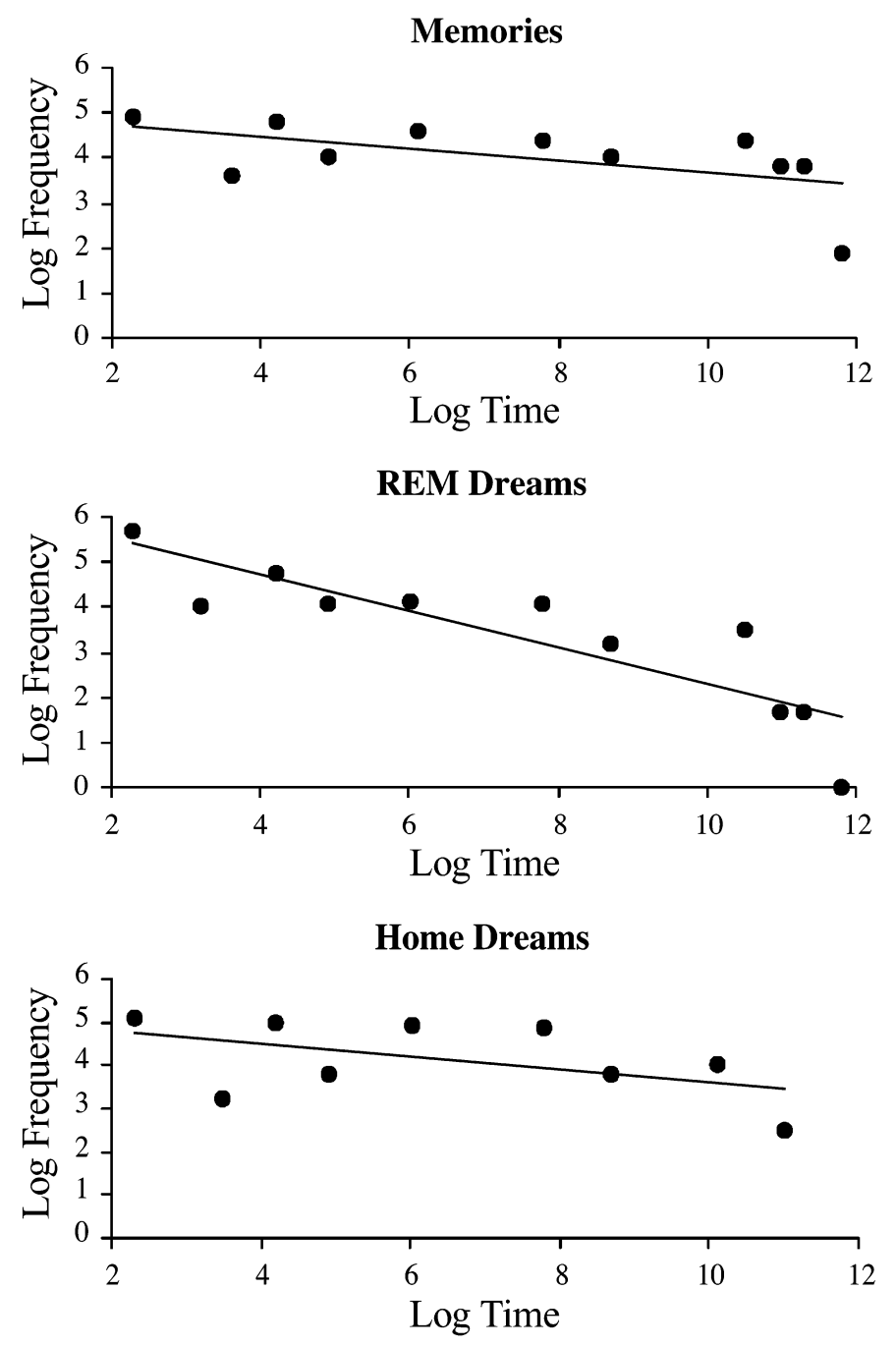

Figure 1. Log of temporal bin frequency as a function of log of temporal bin in hours ago for memories, REM dreams, and home dreams of the younger participants. Every change of one unit is a change of a factor of 10 .

$(M=5.73, S D=5.91)$. Both "this evening" and "yesterday" are day-residue categories for dreams.

The same logarithmic scale method previously described was used to test the prediction of a monotonically decreasing power function for the past 30 years in the case of the older adults. Figure 2 is a plot of the temporal bin frequency as a function of the log of the bin marked in hours ago for memories, REM dreams, and home dreams for the first 30 years of the older participants. Again, the distributions decreased as a function of remoteness with slopes of $-0.07,-0.26$, and -0.19 and intercepts of 4.43, 5.27, and 5.51 for the memories, REM dreams, and home dreams, respectively.

To better visualize the presence of a cubic trend, the distribution of memories and dreams (laboratory and home dream data combined) in terms of the person's "age at time of original experience" is presented in Figure 3. To maximize the reminiscence bump, the data from the category "over 60 years old" are not shown. Note that the memory and dream data for this category are $65.33 \%$ and $84.63 \%$, respectively. It can be seen in Figure 3 that for memories and dreams, there is a high frequency of temporal references dating back to the last 10-20 years, decreasing with increasing remoteness (experiences that took place when participants were 50 and older in terms of their chronological age). Subsequent proportions diminish, followed by a resurgence of references dating to experiences that took place when the participants were adolescents (10-19 years old, for both dreams and memories) and young adults (20-29 years old, for dreams only). This resurgence is followed by a decrease, in both memories and dreams, of references 

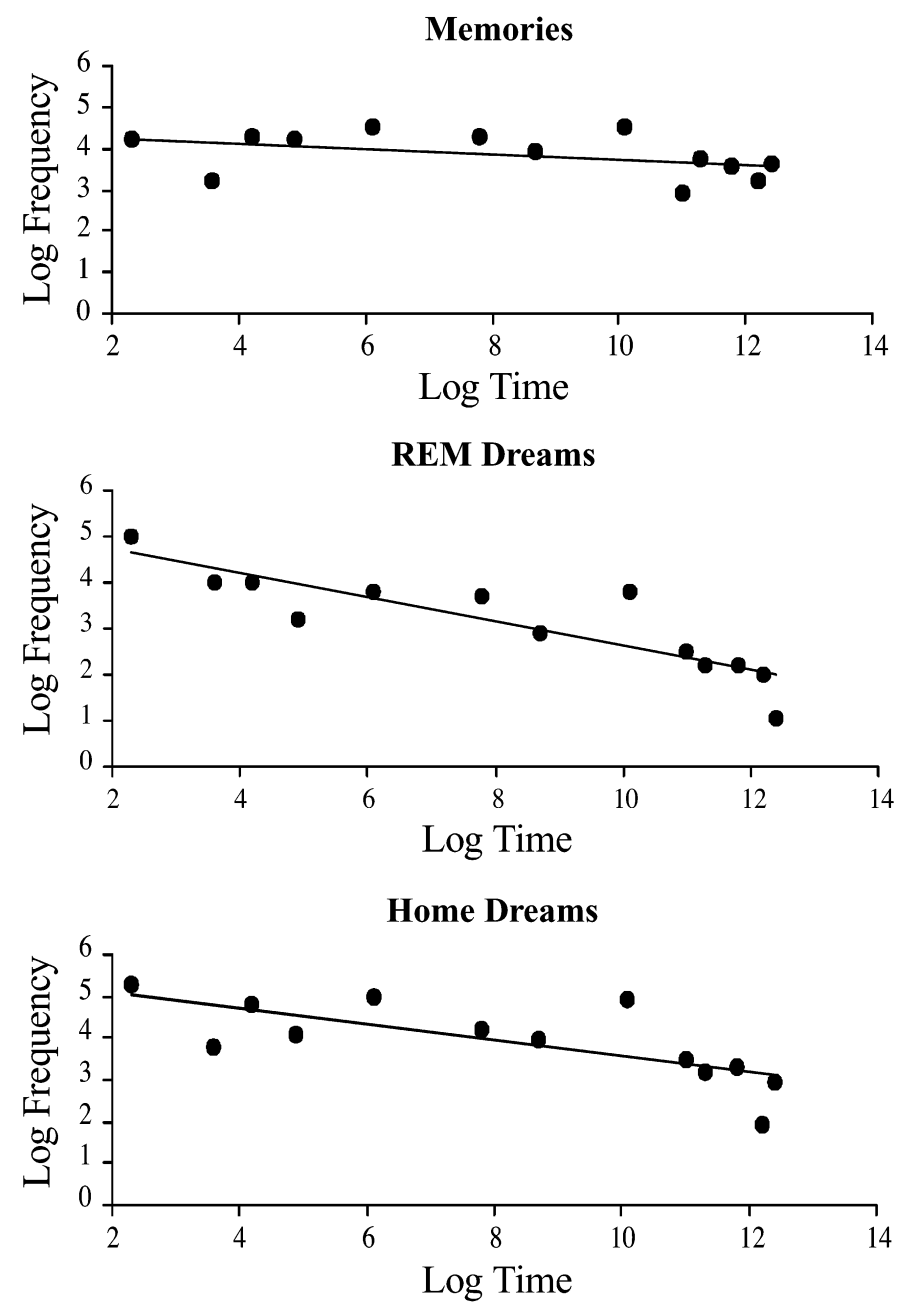

Figure 2. Log of temporal bin frequency as a function of $\log$ of temporal bin in hours ago for memories, REM dreams, and home dreams for the first 30 years of the older participants.

dating to when the participants were children (less than 10 years old).

To determine the presence of a significant cubic trend, contrast analyses were performed on the proportions of memories and dream temporal references (laboratory and home dreams combined) distributed in terms of the participants' age at the time of the original experience. The "over 60 years old" category was not included in the analyses because the high proportion affected the validity of the contrast analyses. For the distribution of autobiographical memories, the contrast analysis $(5,-7,-4$, $4,7,-5)$ indicated a significant cubic trend $[F(1,29)=$ 9.68, $\left.M S_{\mathrm{e}}=37.92, p<.001\right]$. For the distribution of dream temporal references, the same contrast analysis also indicated a significant cubic trend $[F(1,29)=5.88$, $\left.M S_{\mathrm{e}}=21.21, p<.02\right]$.

As for the childhood amnesia component in the older group, although the distribution of temporal references for both memories and dreams decreased following the resurgence observed in the "10-19 years old" decade, the overall distribution of references for dreams and memories differed in that the "less than 10 years old" decade contained the lowest proportion of references in the case of dreams but not in the case of memories. In fact, for memories, the "less than 10 years old" decade had the third-highest proportion out of six temporal bins. Two memories dating to when the participants were less than 3 years old were identified. In REM dreams, two temporal references were identified as dating to the age of 6 . In home dreams, one temporal reference was identified as dating to the age of 4 .

\section{DISCUSSION}

These results confirm the already well-documented observation that for young adults, and for the most recent 30 years in the case of older adults, the distribution of temporal references for memories follows a monoto- 


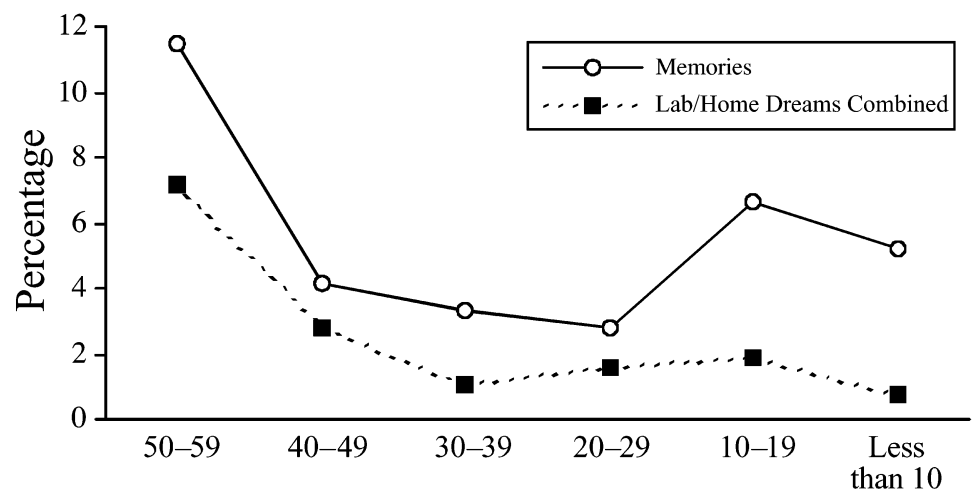

Age at Time of Original Experience

Figure 3. Distribution of memories and dreams (laboratory and home dream data combined) in terms of the person's age at the time of original experience. The data from the category "over 60 years old" are not shown to allow for better visualization of the reminiscence bump. The data for the "over 60 years old" category are $65.33 \%$ and $84.63 \%$ for memories and dreams, respectively.

nically decreasing power function. Our findings also confirm that this is the case for temporal references identified in dreams.

Some differences between the two types of references emerged, however. Of interest is the fact that the younger participants reported significantly fewer memories in the very recent temporal categories ("in the evening" and "yesterday") compared with temporal references identified in REM dreams, and significantly fewer memories in the "this evening" category compared with temporal references identified in home dreams. This suggests that, compared with autobiographical memories generated during the waking state, the elaboration of dreams was more influenced by mnemonic sources of the preceding day (day-residue type sources). Although this may be due to dream elaboration mechanisms, it may also be attributed to methodology. We recognize that the two memory tasks were different. In the case of autobiographical memory, participants responded to a stimulus, whereas in the case of dreams, spontaneous dream narratives were used. In addition, the temporal references were identified and dated in the morning, immediately following dream data collection, at a time when the experiences of the prior evening were still fresh in participants' memory.

As predicted, the young adults identified very few temporal references dating back to early childhood in both memories and in dreams, and none of them explicitly identified either memories or dream temporal references dating before the age of 5, thus supporting the phenomenon of childhood amnesia (Crovitz \& Harvey, 1979; Crovitz, Harvey, \& McKee, 1980; Rubin et al., 1986). Interestingly, more childhood memories seemed to be identified in the older group in comparison with the younger group.

The major prediction for the older group was confirmed: The distributions of dream temporal references and autobiographical memories followed similar cubic trends. This result fits the distribution model of autobiographical memories proposed by Rubin, Rahhal, and Poon (1998). The most important findings are the recrudescence of memories within the 10-19 decade for memories and within the 10-19 and 20-29 decades for dreams. These results replicate a robust finding in autobiographical memory research. Indeed, a number of studies have consistently reported a disproportionately higher number of memories from adolescence and/or early adulthood in older participants (Rubin et al., 1998; Rubin et al., 1986).

In light of Foulkes's (1985) proposition that the cognitive organization of dreams reflects the cognitive organization of the waking state, we suggest that the same cognitive processes postulated to explain the reminiscence bump in autobiographical memory (Rubin et al., 1998) also explain the same bump in dreams. Indeed, memories of adolescence/young adulthood may be more easily and readily retrieved because they were initially stored in periods characterized by rapid personal change, openness to new information on the world, and exposure to much novelty. Variables of novelty and intensive learning would enhance mnemonic performance via an increase in cognitive efforts and minimal proactive interference. Mnemonic elements would thus profit from these memory-enhancing processes and become rich in storage strength, availability, and associative frequency for both waking memory and dream elaboration.

During wakefulness, we have all experienced the phenomena by which we associate a second memory to a first one, then a third to the second, then a fourth to the third, and so on. During sleep, it is suggested (Klinger, 1971) that dream-production mechanisms engage in this same type of associative activity when constructing a dream: An object is associated to a character, then an activity is associated to the object/character duo, then an event is associated to the object/character/activity trio and so forth. If the potential for "associative frequency" 
of mnemonic elements encoded during adolescence and early adulthood is indeed high, we would expect these elements to be retrieved more often by dream production mechanisms.

Rubin et al. (1998) also proposed to account for the reminiscence bump on the basis of identity formation. They referred to the notion that adolescence and young adulthood are critical periods (Erickson, 1950; Fitzgerald, 1988) and that many memories from these periods are retrieved more often for the purpose of being inserted into one's identity narrative (Elnick, Margrett, Fitzgerald, \& Labouvie-Vief, 1999). Reminiscence activities or more specifically, the process of "life review" (Butler, 1963) are examples of this phenomenon.

The need to constitute and reconstitute one's identity at different life periods, and more so in late adulthood, would explain the more frequent memories of adolescence and early adulthood, a characteristic life period of identity development. Our results suggest that adolescence and early adulthood memories would be more frequently retrieved in waking life, thus becoming more easily retrievable and more often found in dreams. However, it should be noted that in this study, the participants indicated that to the best of their knowledge, they had not thought of or talked about the different elements experienced in their dreams since the time of the original experience, implying that these had not been the object of reminiscence in the waking state. Indeed, the participants may have simply forgotten that they had thought of or talked about such elements since the time of the original experience. It would be interesting to examine the profile of references that were identified as having been recently thought of or talked about, and to trace the life period to which they refer in terms of the last time seen or experienced in reality.

Emotions are also to be considered in the mnemonic retrieval process and in dream formation. Since memories dating back to adolescence/early adulthood are often affect laden, emotions experienced in dreams may also hold a high potential for associative frequency and thus stimulate the retrieval of other mnemonic elements within dream formation processes.

A caveat to these explanations of the presence of the reminiscence bump in dreams is a possible methodological artifact. In the morning, our participants spent about 3 consecutive hours identifying temporal references in their dreams. In this process, a number of mnemonic elements or memories might have been activated, creating landmarks to different periods of the participants' lives and potentially guiding subsequent retrievals to the same epochs. It is equally important to note that the dream temporal references were dated during the waking state, under conscious awareness. Therefore, the distribution of dream temporal references may resemble that of waking memory simply because both were dated during the waking state, when the same psychophysiological and cognitive factors influencing autobiographical memory functioning prevailed.
In conclusion, this study bridged two research areas that until now have remained apart: dreaming and autobiographical memory. It represents the first test of a cognitive model of autobiographical memory applied to dreaming and brings to light, as reported for autobiographical memories, the fact that very remote elements identified in the dreams of older adults refer preferentially to the same periods of adolescence and/or early adulthood. This finding supports the hypothesis of continuity in cognitive organization between autobiographical memories in the waking state and mnemonic elements in dreams.

\section{REFERENCES}

Beck, A. T., Ward, C. H., Mendleson, M., Mock, J., \& Erbaugh, J. (1961). An inventory for measuring depression. Archives of General Psychiatry, 4, 561-571.

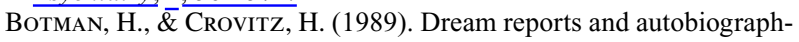 ical memory. Imagination, Cognition \& Personality, 9, 213-224.

ButLer, R. N. (1963). The life review: An interpretation of reminiscence in the aged. Psychiatry: Journal for the Study of Interpersonal Processes, 26, 65-76.

CAVAllero, C., \& Foulkes, D. (1993). Dreaming as cognition. London: Harvester Wheatsheaf.

Crovitz, H. F., \& Harvey, M. T. (1979). Early childhood amnesia: A quantitative study with implications for the study of retrograde amnesia after brain injury. Cortex, 15, 331-335.

Crovitz, H. F., Harvey, M. T., \& McKee, D. C. (1980). Selecting retrieval cues for early childhood amnesia: Implications for the study of shrinking retrograde amnesia. Cortex, 16, 305-310.

DAVIDSON, J., \& Kelsey, B. (1987). Incorporations of recent events in dreams. Perceptual \& Motor Skills, 65, 114.

Domнoff, G. W. (2003). The scientific study of dreams. Washington, DC: American Psychological Association.

Elnick, A. B., Margrett, J., Fitzgerald, J., \& Labouvie-Vief, G. (1999). Benchmark memories in adulthood: Central domains and predictors of their frequency. Journal of Adult Development, 6, 45-59.

EPSTEIN, A. (1985). The waking event-dream interval. American Journal of Psychiatry, 142, 123-124.

ERICKSON, E. H. (1950). Childhood and society. New York: Norton.

FitzGERALD, J. M. (1988). Vivid memories and the reminiscence phenomenon: The role of the self narrative. Human Development, 31, 261-273.

FitzGerald, J. M., \& LaWrence, R. (1984). Autobiographical memory across the life-span. Journal of Gerontology, 39, 692-698.

FoulKes, D. (1985). Dreaming: A cognitive-psychological analysis. Hillsdale, NJ: Erlbaum.

Franklin, H. C., \& Holding, D. H. (1977). Personal memories at different ages. Quarterly Journal of Experimental Psychology, 29, 527-532.

GARDINER, J. M., \& JAVA, R. I. (1991). Forgetting in recognition memory with and without recollective experience. Memory \& Cognition, 19, 617-623.

GiAMBra, L., JUnG, R., \& Grodsky, A. (1996). Age changes in dream recall in adulthood. Dreaming, 6, 17-31.

Hartmann, E. (1968). The day-residue: Time distribution of waking events. Psychophysiology, 5, 222.

Hobson, J. A. (1988). The dreaming brain. New York: Basic Books. Jouvet, M. (1979). Mémoire et "cerveau dédoublé" au cours du rêve à propos de 130 souvenirs de rêve. L'Année du Practicien, 29, 27-32.

KLINGER, E. (1971). The structure and function of fantasy. New York: Wiley.

Marquardt, C. J. G., Bonato, R. A., \& Hoffman, R. F. (1996). An empirical investigation into the day-residue and dream-lag effects. Dreaming, 6, 57-65.

McCormack, P. D. (1979). Autobiographical memory in the aged. Canadian Journal of Psychology, 33, 118-124.

NiELSEN, T., \& Powell, R. (1992). The day-residue and dream-lag ef- 
fects: A literature review and limited replication of the two temporal effects in dream formation. Dreaming, 2, 67-77.

Paivio, A., Yuille, J., \& Madigan, S. (1968). Concreteness, imagery, and meaningfulness values for 925 nouns. Journal of Experimental Psychology, 76 (1, Pt. 2), 1-25.

Rechtschaffen, A., \& Kales, A. (1968). A manual of standardized terminology, techniques and scoring system for sleep stages of human subjects. Washington, DC: Public Health Service, U.S. Government Printing Office.

RubiN, D. C. (1982). On the retention function for autobiographical memory. Journal of Verbal Learning \& Verbal Behavior, 21, 21-38.

Rubin, D. C., RahHal, T. A., \& Poon, L. W. (1998). Things learned in early adulthood are remembered best. Memory \& Cognition, 26, 3-19.
Rubin, D. C., Wetzler, S. E., \& Nebes, R. D. (1986). Autobiographical memory across the life-span. In D. C. Rubin (Ed.), Autobiographical memory (pp. 202-221). Cambridge: Cambridge University Press.

Stuss, D., Meiran, N., Guzman, A., Lafleche, G., \& Willmer, J. (1996). Do long tests yield a more accurate diagnosis of dementia than short tests? Archives of Neurology, 53, 1033-1039.

Verdone, P. (1965). Temporal reference of manifest dream content. Perceptual \& Motor Skills, 20, 262-271.

Winget, C., Kramer, M., \& Whitman, R. M. (1970). The relationship of socioeconomic status and race of respondent to normative dream content. Psychophysiology, 7, 325.

Zola-Morgan, S., Cohen, N. J., \& SQuire, L. R. (1983). Recall of remote episodic memory in amnesia. Neuropsychologia, 21, 487-500.

(Manuscript received November 28, 2000;

revision accepted for publication September 24, 2004.) 